\title{
EXTREMELY RED OBJECTS IN THE LOCKMAN HOLE
}

\author{
G. Wilson, ${ }^{1}$ J.-S. Huang, ${ }^{2}$ P. G. Pérez-González, ${ }^{3}$ E. Egami, ${ }^{3}$ R. J. Ivison, ${ }^{4}$ J. R. Rigby, ${ }^{3}$ A. Alonso-Herrero, ${ }^{3}$ P. Barmby, ${ }^{2}$ \\ H. Dole, ${ }^{3,5}$ G. G. Fazio, ${ }^{2}$ E. Le Floc' ${ }^{3}{ }^{3}$ C. Papovich, ${ }^{3}$ D. Rigopoulou, ${ }^{6}$ L. Bai, ${ }^{3}$ C. W. Engelbracht, ${ }^{3}$ D. Frayer, ${ }^{1}$ K. D. Gordon, ${ }^{3}$ \\ D. C. Hines, ${ }^{3}$ K. A. Misselt, ${ }^{3}$ S. Miyazaki, ${ }^{7}$ J. E. Morrison, ${ }^{3}$ G. H. Rieke, ${ }^{3}$ M. J. Rieke, ${ }^{3}$ and J. Surace ${ }^{1}$ \\ Received 2004 March 26; accepted 2004 May 21
}

\begin{abstract}
We investigate extremely red objects (EROs) using near- and mid-infrared observations in five passbands (3.6 to $24 \mu \mathrm{m}$ ) obtained from the Spitzer Space Telescope, and deep ground-based $R$ and $K$ imaging. The great sensitivity of the Infrared Array Camera (IRAC) camera allows us to detect 64 EROs (a surface density of $\left.2.90 \pm 0.36 \operatorname{arcmin}^{-2} ;[3.6]_{\mathrm{AB}}<23.7\right)$ in only 12 minutes of IRAC exposure time, by means of an $R-[3.6]$ color cut (analogous to the traditional red $R-K$ cut). A pure infrared $K-[3.6]$ red cut detects a somewhat different population and may be more effective at selecting $z>1.3$ EROs. We find $\sim 17 \%$ of all galaxies detected by IRAC at 3.6 or $4.5 \mu \mathrm{m}$ to be EROs. These percentages rise to about $40 \%$ at $5.8 \mu \mathrm{m}$, and about $60 \%$ at $8.0 \mu \mathrm{m}$. We utilize the spectral bump at $1.6 \mu \mathrm{m}$ to divide the EROs into broad redshift slices using only near-infrared colors $(2.2 / 3.6 / 4.5 \mu \mathrm{m})$. We conclude that two-thirds of all EROs lie at redshift $z>1.3$. Detections at $24 \mu \mathrm{m}$ imply that $a t$ least $11 \%$ of $0.6<z<1.3$ EROs and at least $22 \%$ of $z>1.3$ EROs are dusty star-forming galaxies.

Subject headings: cosmology: observations — galaxies: evolution — galaxies: high-redshift — galaxies: photometry — galaxies: starburst — infrared: galaxies
\end{abstract}

\section{INTRODUCTION}

First discovered in the late 1980s (Elston et al. 1988) extremely red objects (EROs) are defined by their very red optical/near-infrared colors. Their faintness makes them difficult to classify spectroscopically or morphologically. The redness of their color constrains these galaxies to be either early-type (elliptical and S0) galaxies in the redshift range $1<z<2$, or luminous dusty late-type galaxies at high redshift.

These two classes of EROs may possibly represent different phases in the formation and evolution of present-day massive elliptical galaxies. However, the two rival scenarios of galaxy formation (pure luminosity evolution and hierarchical clustering) predict very different formation epochs for such galaxies. Current semianalytic models appear to underpredict the number of EROs at $z>1$ (Cimatti et al. 2002b; Somerville et al. 2003). EROs, therefore, are of particular interest to cosmologists, since studying their properties, e.g., number density, colors, redshift distribution, and star formation rates, can provide crucial constraints on contemporary galaxy evolution models.

Some large fraction of EROs are undoubtedly the progenitors of present-day early-type galaxies. It has been known since at least Davis \& Geller (1976) that early-type galaxies cluster more strongly than late-type galaxies. EROs display extremely strong clustering (Roche et al. 2002; Daddi et al.

\footnotetext{
1 Spitzer Science Center, California Institute of Technology, MC 220-6, Pasadena, CA 91125; gillian@ipac.caltech.edu.

${ }^{2}$ Harvard-Smithsonian Center for Astrophysics, 60 Garden Street, Cambridge, MA 02138.

${ }^{3}$ Steward Observatory, University of Arizona, Tucson, AZ 85721.

4 Astronomy Technology Centre, Royal Observatory, Blackford Hill, Edinburgh EH9 3HJ, UK.

${ }_{5}^{5}$ Institut d'Astrophysique Spatiale, bat 121, Universiteé Paris Sud, F-91405 Orsay Cedex, France.

6 Department of Astrophysics, Oxford University, Keble Road, Oxford, OX1 3RH, UK.

7 Subaru Telescope, National Astronomical Observatory of Japan, 650 North A'ohoku Place, Hilo, HI 96720.
}

2003), providing strong support to the theory that EROs are massive ellipticals. However, contrarily, many EROs appear to be highly obscured starburst galaxies (Graham \& Dey 1996). Studies of their morphologies (Yan \& Thompson 2003; Moustakas et al. 2004) suggest that the population consists of a mixture of early- and late-type galaxies in approximately equal measure.

Valiant attempts have been made to use optical-near-infrared colors (in various combinations of RIJHK) to distinguish between passive and star-forming dusty EROs (Pozzetti \& Mannucci 2000; Franx et al. 2003; Bergström \& Wiklind 2004). This method, however, requires extremely precise photometry and is not yet well tested (Mannucci et al. 2002; Smail et al. 2002). In this paper we show how mid-infrared data from the Spitzer Space Telescope can easily identify the dusty starburst population. In $\S 2$ we describe the data set. In $\S 3$ we relate the Spitzer data to the traditional $R-K$ color cut. We introduce new $R-[3.6]$ and $K-[3.6]$ color cuts. We use the $1.6 \mu \mathrm{m}$ bump (John 1988) to place broad redshift constraints on the selected EROs. We then discuss the interpretation of matched IRAC, MIPS $(24 \mu \mathrm{m})$, and SCUBA (850 $\mu \mathrm{m})$ detections. We conclude in $\S 4$.

\section{OBSERVATIONS}

\subsection{Spitzer Space Telescope and Ancillary Data}

The observations described here were performed as part of the Spitzer Space Telescope Early Release Observation program. The portion of the Lockman Hole field that has complete (3.6-24 $\mu \mathrm{m})$ Spitzer coverage spans an area of $4.7 \times 4.7$ centered on R.A. $=10^{\mathrm{h}} 51^{\mathrm{m}} 56.0$, decl. $=57^{\circ} 25^{\prime} 32^{\prime \prime} .0(\mathrm{~J} 2000)$. Full descriptions of the Infrared Array Camera (IRAC) may be found in Fazio et al. (2004), and the Multiband Imaging Photometer for Spitzer (MIPS) in Rieke et al. (2004). The Lockman Hole data reduction procedures and observations are discussed in Egami et al. (2004), Huang et al. (2004), Le Floc'h et al. (2004), and Papovich et al. (2004). An analysis of Spitzer counterparts to XMM-Newton, SCUBA, and 
TABLE 1

Flux Limits

\begin{tabular}{|c|c|c|c|c|c|c|}
\hline Passband & Galaxies & $(R-[3.6])_{\mathrm{AB}}>4.0$ & $\%$ ERO & $(K-[3.6])_{\mathrm{AB}}>0.7$ & $\%$ ERO & $5 \sigma$ Mag Limit (AB) \\
\hline 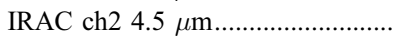 & 403 & 64 & 15.9 & 70 & 17.4 & 23.77 \\
\hline 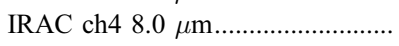 & 80 & 35 & 43.8 & 46 & 57.5 & 21.68 \\
\hline MIPS ch1 $24.0 \mu \mathrm{m}$ & 32 & 11 & 34.4 & 13 & 40.6 & 18.15 \\
\hline
\end{tabular}

MAMBO sources is presented in Alonso-Herrero et al. (2004), Egami et al. (2004), Ivison et al. (2004) and Serjeant et al. (2004). The number of galaxies detected by Spitzer with $\mathrm{S} / \mathrm{N}>5$, and the corresponding magnitude limits for each passband, are shown in Table 1.

In addition to the Spitzer observations, we utilized principally $R$ data $\left(R_{\mathrm{AB}}=27.3 ; 5 \sigma\right)$ from the Suprime camera on the Subaru Telescope (Miyazaki et al. 2002), $K$ data $\left(K_{\mathrm{AB}}=\right.$ 23.3; $5 \sigma$ ) from the Omega-Prime camera on the Calar Alto Telescope (Huang et al. 2001), SCUBA data from the James Clerk Maxwell Telescope (JCMT) (Scott et al. 2002), and $X M M$-Newton data (Hasinger et al. 2001) for this study.

Stars were removed from our catalogs based on a combination of their morphologies in the deep $R$-band image and their location in a $V-I$ versus $I-K$ color-color diagram (Huang et al. 1997; Wilson 2003). Our final catalog, containing sources detected at both 3.6 and $4.5 \mu \mathrm{m}$ (the two most sensitive IRAC channels), numbers 329 objects (further details may be found in Huang et al. 2004).

\section{EXTREMELY RED OBJECT SELECTION}

\subsection{The $R-K$ Sample}

An ERO is traditionally defined as an object satisfying $R-K>5.0$ in the Vega magnitude system. Here we choose to present all magnitudes in the $\mathrm{AB}$ system. ${ }^{8}$ Comparing our $R-K$ selected sample with published results (Table 2), we observe an ERO surface density of $1.62 \pm 0.27,2.26 \pm 0.32$ and $2.76 \pm 0.35 \mathrm{arcmin}^{-2}$ to $K_{\mathrm{AB}}<21.9,22.4$ and 22.9 (total number of galaxies in parentheses). These values compare well to the surface densities of $1.50 \pm 0.17$ and $1.69 \pm$ $0.10 \mathrm{arcmin}^{-2}$ found by Cimatti et al. (2002a) and Moustakas et al. (2004), respectively. The agreement with Roche et al. (2002) is poorer. Note, however, that the uncertainties in Table 2 are Poissonian and hence underestimates, because

\footnotetext{
${ }^{8}$ Since $R_{\mathrm{Vega}}=R_{\mathrm{AB}}-0.19$ and $K_{\mathrm{Vega}}=K_{\mathrm{AB}}-1.89,(R-K)_{\mathrm{AB}}>3.3$ corresponds to $(R-K)_{\mathrm{Vega}}>5.0$.
}

cosmic variance is not included (Somerville et al. 2004). Using a redder $(R-K)_{\mathrm{AB}}>3.6$ cut [equivalent to $(R-K)_{\text {Vega }}>$ $5.3]$, the ERO surface densities we find also agree reasonably well with the values obtained by Smail et al. (2002) and Moustakas et al. (2004).

In $\S 3.2$ we investigate how IRAC $3.6 \mu \mathrm{m}$ data can be used to select EROs. Firstly, we speculate on the different nature of objects likely to be selected by $R-K, R-[3.6]$, and $K-[3.6]$ red color cuts. One might expect a red $R-[3.6]$ cut to select a very similar galaxy sample as a red $R-K$ cut, i.e., elliptical galaxies at $z=1-2$ and very dusty star-forming galaxies at high redshift. Therefore, either an $R-K$ or an $R-[3.6]$ selected sample would be likely to contain galaxies with a rather heterogeneous redshift distribution. A traditional $R-K$ red selection might bias the sample slightly against high-redshift objects compared to an $R-$ [3.6] selection because the $K$ passband samples rest-frame optical wavelengths at lower redshift than [3.6]. Thus, any small amount of star formation might cause a galaxy to appear blue and preclude it from being included in the $R-K$ sample, while it might still be included in the $R-[3.6]$ sample.

Historically, e.g., Cowie et al. (1990), it was thought that selecting galaxies with the reddest infrared colors might preferentially select galaxies at the highest redshifts. A $K-[3.6]$ color provides an extra independent measurement of the slope of a galaxy's spectral energy distribution (SED) at longer wavelength than the traditional $R-K$ measurement. Therefore, selecting red galaxies based upon their infrared $K-[3.6]$ color might well result in a sample of galaxies with a more homogeneous redshift distribution than an $R-K$ cut, because pure-infrared selection is almost insensitive to dust extinction and instead measures the slope of the spectral energy distribution, which is similar for all galaxy types at any given redshift (with the exception of AGNs).

\subsection{The $R-[3.6]$ and $K-[3.6]$ Samples}

The Spitzer color cut that corresponds most closely to a conventional $R-K$ selection is $R-[3.6]$. We determined

TABLE 2

$R-K$ Selected ERo Surface Densities

\begin{tabular}{|c|c|c|c|c|c|c|}
\hline \multirow[b]{2}{*}{ REFERENCE } & \multicolumn{3}{|c|}{$(R-K)_{\mathrm{AB}}>3.3 ;(R-K)_{\mathrm{Vega}}>5.0$} & \multicolumn{3}{|c|}{$(R-K)_{\mathrm{AB}}>3.6 ;(R-K)_{\mathrm{Vega}}>5.3$} \\
\hline & $\begin{array}{c}K<21.9 \\
\left(K_{\text {Vega }}<20.0\right)\end{array}$ & $\begin{array}{c}K<22.4 \\
\left(K_{\text {Vega }}<20.5\right)\end{array}$ & $\begin{array}{c}K<22.9 \\
\left(K_{\text {Vega }}<21.0\right)\end{array}$ & $\begin{array}{c}K<21.9 \\
\left(K_{\mathrm{Vega}}<20.0\right)\end{array}$ & $\begin{array}{c}K<22.4 \\
\left(K_{\text {Vega }}<20.5\right)\end{array}$ & $\begin{array}{c}K<22.9 \\
\left(K_{\text {Vega }}<21.0\right)\end{array}$ \\
\hline 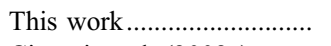 & $1.62 \pm 0.27(36)$ & $2.26 \pm 0.32(50)$ & $2.76 \pm 0.35(61)$ & $1.13 \pm 0.23(25)$ & $1.40 \pm 0.25(31)$ & $1.77 \pm 0.28(39)$ \\
\hline Cimatti et al. (2002a).......... & $1.50 \pm 0.17$ & $\ldots$ & $\ldots$ & $\ldots$ & $\ldots$ & $\ldots$ \\
\hline Moustakas et al. (2004) ...... & $1.69 \pm 0.10$ & $\ldots$ & $\ldots$ & $1.13 \pm 0.08$ & $\ldots$ & $\ldots$ \\
\hline
\end{tabular}

Note.-The units are $\operatorname{arcmin}^{-2}$, the uncertainties are Poissonian. 
TABLE 3

$R-[3.6]$ Selected ERO Samples

\begin{tabular}{|c|c|c|c|c|c|}
\hline$z$ & $(R-[3.6])_{\mathrm{AB}}>4.0$ & $N$ & $24 \mu \mathrm{m}$ & $\%$ & SCUBA \\
\hline All & & 64 & 11 & 17.2 & 4 \\
\hline $0.6<z<1.3 \ldots \ldots \ldots \ldots \ldots$ & $(K-[3.6])_{\mathrm{AB}}>0$ and $([3.6]-[4.5])_{\mathrm{AB}}<0$ & 18 & 2 & 11.1 & 0 \\
\hline$z>1.3 \ldots \ldots \ldots \ldots \ldots \ldots \ldots \ldots$ & $(K-[3.6])_{\mathrm{AB}}>0$ and $([3.6]-[4.5])_{\mathrm{AB}}>0$ & 44 & 9 & 20.5 & 4 \\
\hline
\end{tabular}

empirically that galaxies with red $\mathrm{AB}$ color of $R-[3.6]>4.0$ correspond to those with $R-K>3.3$. (We did this by calculating the mode $[=3.9]$ of the color of all galaxies in the $R-K>3.3$ sample. We then subtracted the same offset of 0.6 from the mode of the $R-$ [3.6] color.) We refer to galaxies selected with color $R-[3.6]>4.0$ as our $R-$ [3.6] selected sample. As shown in Tables 1 and 3, 64 objects satisfy the $R-$ [3.6] color cut.

There are 59 galaxies in common between the $R-K(72)$ and $R-[3.6]$ (64) selected red samples. Hence, as expected (§ 3.1), we conclude that these two color cuts result in very similar samples. (We also experimented with IRAC channel 2 color cuts, i.e., $R-[4.5]$ and $K-[4.5]$, but found these to give essentially the same results as the $3.6 \mu \mathrm{m}$ cuts, albeit with slightly increased scatter.)

One great advantage of having observations at 3.6 and $4.5 \mu \mathrm{m}$ is that one can utilize $K /[3.6] /[4.5]$ relative colors to place additional redshift constraints on the galaxies. In the rest-frame near-infrared, the most important spectral feature is the $1.6 \mu \mathrm{m}$ bump, universal to all spectral types of galaxies, except AGN-dominated SEDs, and long considered as a photometric redshift indicator (Sawicki 2002). At $z=0.6$, the bump lies midway between the $K$ and $3.6 \mu \mathrm{m}$ passbands, and so a galaxy at that redshift would be expected to have a neutral $K-[3.6]=0$ color. At higher redshift, the bump moves further into the $3.6 \mu \mathrm{m}$ band, causing galaxies to have increasingly red, $K-[3.6]>0$, colors. At $z=1.3$, the $1.6 \mu \mathrm{m}$ bump falls midway between the 3.6 and $4.5 \mu \mathrm{m}$ passbands. By a similar argument, a galaxy with $\mathrm{AB}$ color $[3.6]-[4.5]>0$ would be expected to lie at a redshift $z>1.3$ (see also Huang et al. 2004).

Figure 1 shows a $K-[3.6]$ versus [3.6] $-[4.5]$ color-color diagram. The open and filled blue (and open and filled black) circles denote the 64 objects selected as EROs on the basis of their red $R-[3.6]$ colors. As shown in Table 3, 18 galaxies were found to have $K-[3.6]>0$ and [3.6] $-[4.5]<0$ color (thus likely lying in the redshift range $0.6<z<1.3$ ) and 44 galaxies were found to have $K-[3.6]>0$ and [3.6]$[4.5]>0$ (thus likely lying in the redshift range $z>1.3$ ). The two galaxies with color $K-[3.6]<0$ are likely dusty local galaxies.

By definition, once two out of the three possible $R / K /[3.6]$ color combinations have been chosen, the third is also determined. $\mathrm{An} \mathrm{AB}$ color of $K-[3.6]=0.7$ corresponds to an $R-K=3.3$ and a $R-[3.6]=4.0$ color. The open and filled green (and open and filled black) circles in Figure 1 denote the 73 objects that satisfied the $K-[3.6]$ color cut (Table 4). Our sample contained $21 \mathrm{~K}-$ [3.6] selected EROs with $K-$ [3.6] $>0$ and [3.6] $-[4.5]<0(0.6<z<1.3)$, and 49 galaxies with $K-[3.6]>0$ and [3.6] $-[4.5]>0(z>1.3)$.

\subsection{Discussion}

As expected, although both the $R-$ [3.6] cut and $K-$ [3.6] do indeed select many of the same EROs, they do not select exactly the same galaxies. The open and filled black circles in
Figure 1 show EROs in common, i.e., galaxies that would be identified as EROs by either color cut. There are 40 galaxies in common between the $K-$ [3.6] (70) and $R-$ [3.6] (64) selected samples, and 35 in common between the $K-[3.6]$ and $R-K(72)$ selected samples (totals in parentheses). Although our sample is small, these results do appear to support the scenario proposed in $\S 3.1$. As expected, most of the galaxies selected by both the $R-[3.6]$ and $K-[3.6]$ criteria appear to lie at high redshift.

Regardless of whether one employs a $R-[3.6]$ cut or a $K-$ [3.6] color cut, it is clear that a very high percentage of all galaxies detected by IRAC would be classified as extremely red objects. From Table 1, about $17 \%$ of all galaxies detected at 3.6 and $4.5 \mu \mathrm{m}$ are EROs. These percentages rise to about $40 \%$ at $5.8 \mu \mathrm{m}$, and an astonishing $60 \%$ at $8.0 \mu \mathrm{m}$. The smaller percentage (40\%) of EROs detected at $24 \mu \mathrm{m}$ by MIPS is almost certainly due to a combination of the relative

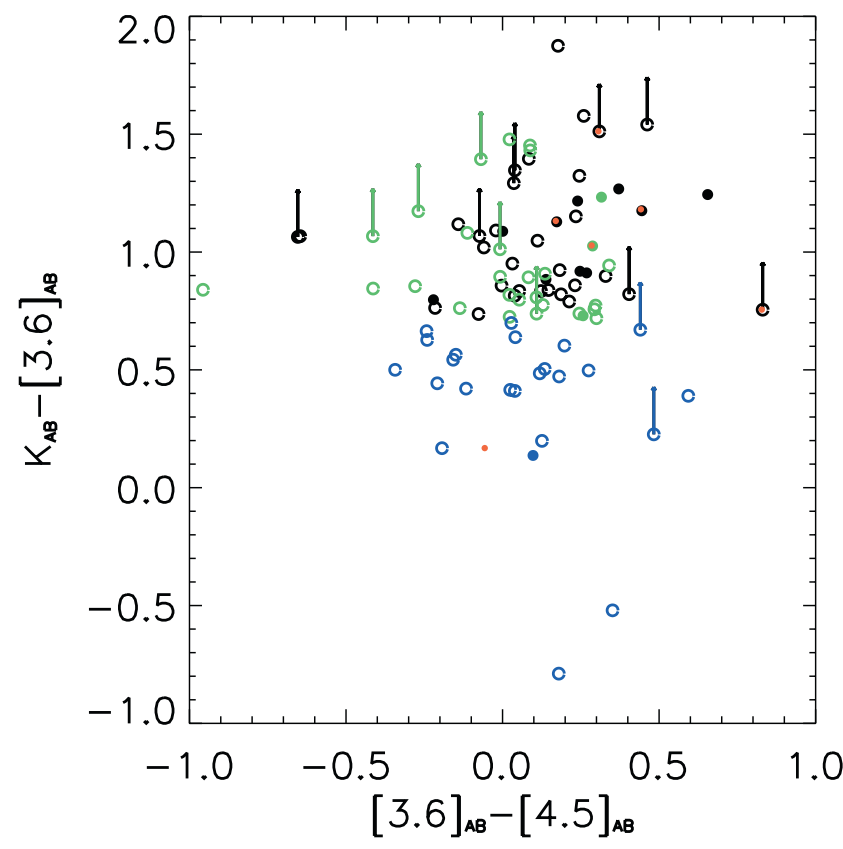

FIG. 1. $-K-[3.6]$ vs. [3.6] $-[4.5]$ color-color diagram for extremely red objects (all magnitudes are $\mathrm{AB}$ ). The arrows indicate galaxies detected in IRAC channel $1(3.6 \mu \mathrm{m})$ but not detected in the $K$ band (and hence having only a lower limit to their $K-[3.6]$ color). The open and filled blue circles denote EROs selected on the basis of their red $R-[3.6]$ colors. Similarly, the open and filled green circles denote EROs selected on the basis of their red $K-[3.6]$ colors. The open and filled black circles show EROs in common, i.e., galaxies that would be identified as EROs by either color cut. Galaxies with $K-[3.6]>0$ and $[3.6]-[4.5]<0$, i.e., occupying the upper left quadrant, likely lie in the redshift range $0.6<z<1.3$. Galaxies with $K-[3.6]>0$ and $[3.6]-[4.5]>0$, i.e., occupying the upper right quadrant, likely lie at redshift $z>1.3$. Note that the agreement between the $R-[3.6]$ and $K-[3.6]$ selected samples is best at $z>1.3$. The blue, green and black filled circles, in each case, show galaxies with a MIPS $24 \mu \mathrm{m}$ detection. Red filled circles denote SCUBA sources (Scott et al. 2002). The two galaxies with color $K-[3.6]<0$ are likely dusty local galaxies. 
TABLE 4

$K-$ [3.6] Selected ERO Samples

\begin{tabular}{|c|c|c|c|c|c|}
\hline$z$ & $(K-[3.6])_{\mathrm{AB}}>0.7$ & $N$ & $24 \mu \mathrm{m}$ & $\%$ & SCUBA \\
\hline All ............................... & & 70 & 13 & 18.6 & 5 \\
\hline $0.6<z<1.3 \ldots \ldots \ldots \ldots$ & $([3.6]-[4.5])_{\mathrm{AB}}>0$ & 21 & 2 & 9.5 & 0 \\
\hline$z>1.3 \ldots \ldots \ldots \ldots \ldots \ldots \ldots$ & $([3.6]-[4.5])_{\mathrm{AB}}>0$ & 49 & 11 & 22.4 & 5 \\
\hline
\end{tabular}

MIPS/IRAC exposure times and the higher background at $24 \mu \mathrm{m}$.

The filled circles in Figure 1 denote galaxies that are also detected (at $>5 \sigma$ ) by MIPS at $24 \mu \mathrm{m}$. All galaxies detected at $24 \mu \mathrm{m}$ have corresponding detections at 5.8 and $8.0 \mu \mathrm{m}$. We interpret a $24 \mu \mathrm{m}$ detection as evidence of a dusty starburst galaxy. We find two (11.1\%) $24 \mu \mathrm{m}$ detections in the redshift range $0.6<z<1.3$ and nine (20.5\%) $24 \mu \mathrm{m}$ detections in the redshift range $z>1.3$ for the $R-$ [3.6] sample. We find two (9.5\%) $24 \mu \mathrm{m}$ detections in the redshift range $0.6<z<1.3$ and $11(22.4 \%) 24 \mu \mathrm{m}$ detections in the redshift range $z>1.3$ for the $K-[3.6]$ sample. Since it is impossible to determine whether the absence of a detection at $24 \mu \mathrm{m}$ is due simply to an intrinsically infrared-faint or distant dusty starburst generating insufficient flux to have been detected within this exposure time, or due to a passively evolving early-type galaxy, our technique effectively places lower limits on the percentage contribution of dusty starbursts to the total ERO population. We find lower limits to the surface densities for the dusty starbursts of more than $0.09 \mathrm{arcmin}^{-2}$ for $0.6<z<1.3$ and more than $0.41 \operatorname{arcmin}^{-2}$ for $z>1.3$ for the red $R-$ [3.6] sample, and similarly, more than $0.09 \mathrm{arcmin}^{-2}$ and more than $0.50 \operatorname{arcmin}^{-2}$ for the $K-[3.6]$ sample.

If one assumes that the local correlations between mid and total $(8-1000 \mu \mathrm{m})$ infrared luminosities (Elbaz et al. 2002, $\S 4.2 .2)$ remain valid at higher redshift, it is possible to infer the minimum luminosity of a galaxy at any given redshift using our $24 \mu \mathrm{m}$ flux limit (Table 1). We conclude that the EROs we select at $0.6<z<1.3$ with $24 \mu \mathrm{m}$ detections could either be starbursts or luminous infrared galaxies (LIRGs), and the EROs we select at $z>1.3$ could be starbursts, LIRGs, or ultraluminous infrared galaxy (ULIRGs). (Interestingly, no $X M M$-Newton-detected galaxies had sufficiently red colors to appear in our $R-$ [3.6] selected ERO sample, whilst one XMMNewton source was selected by the $K-[3.6]$ cut. This suggests that AGNs are generally too blue in color to meet the ERO selection criteria and do not constitute a major contaminant).

There are seven SCUBA sources ( $>3.5 \sigma$ detections) in our field (Scott et al. 2002, see also Egami et al. 2004). Of those seven, IRAC detects six counterparts. The red filled circles in Figure 1 denote the SCUBA sources. One of the sources has close neighbors and so the optical/infrared colors are unreliable. Of the remaining five, all five satisfy our $K-[3.6]$ color selection criteria (four satisfy our $R-$ [3.6] color selection criteria) and three were detected at $24 \mu \mathrm{m}$. Reassuringly, all five are assigned to the $z>1.3$ redshift interval based on their colors, providing verification that our color criteria are indeed selecting galaxies at the appropriate redshift. Matching SCUBA sources to the IRAC EROs in the redshift interval $z>1.3$ is consistent with the interpretation of these galaxies as ultraluminous dusty starbursts at $z \simeq 2.5$ (Chapman et al. 2003).

One additional galaxy, identified by us as an ERO at $z>1.3$, was found to have a spectroscopically confirmed redshift of $z=2.38$ (R. J. Ivison et al. 2004, in preparation). This provides additional confirmation of the accuracy of our redshift assignments. Interestingly, this galaxy is extremely luminous. With a flux of $857 \mu \mathrm{Jy}$, it is the most luminous $24 \mu \mathrm{m}$ detection in the ERO catalogs (the second most luminous galaxy has a flux of $386 \mu \mathrm{Jy}$ ). The nature of this source has not yet been determined. It is likely either a very luminous dusty starburst galaxy or an AGN. Studies of this interesting source are ongoing.

\section{CONCLUSIONS}

In this paper, for the first time, we were able to utilize a new window on the near and mid-infrared universe, using 3.6 to $24 \mu \mathrm{m}$ data from the Spitzer Space Telescope to explore the nature of EROs. We found that the great sensitivity of the IRAC camera allows EROs to be easily detectable. Using an $R-$ [3.6] color cut, we detected 64 EROs (a surface density of $3.03 \pm 0.37 \mathrm{arcmin}^{-2} ;[3.6]_{\mathrm{AB}}<23.7$ ) in only 12 minutes of IRAC exposure time. Although our sample was small, there was evidence that a pure infrared $K-[3.6]$ color cut might be even more effective in finding high-redshift EROs. The $K-$ [3.6] cut identified two $z>1.3$ galaxies with $24 \mu \mathrm{m}$ detections and one SCUBA source not selected by the $R-[3.6]$ color criterion. This caused us to conclude that the pure infrared $K-[3.6]$ color cut may be slightly more effective than the longer baseline $R-[3.6]$ color cut in selecting $z>2$ dusty star-forming galaxies ("the SCUBA population"). Regardless of the subtleties of the Spitzer color cut employed, we found about $17 \%$ of all galaxies detected by IRAC at 3.6 or $4.5 \mu \mathrm{m}$ to be EROs. These percentages rose to about $40 \%$ at $5.8 \mu \mathrm{m}$, and about $60 \%$ at $8.0 \mu \mathrm{m}$.

We concluded that two-thirds of all EROs lie at redshift $z>1$.3. Independent evidence for this conclusion was provided by the existence of six counterparts to our IRAC detections, five SCUBA sources (almost undoubtedly lying at redshifts $2<z<3$ ) and a spectroscopically confirmed redshift of 2.38 for the most luminous MIPS $24 \mu \mathrm{m}$ detection in our ERO catalog.

The existence of detections at $24 \mu \mathrm{m}$ allowed us to place lower limits on the percentage contribution of dusty starbursts to the total ERO population. We concluded that at least $11 \%$ of $0.6<z<1.3$ EROs and at least $22 \%$ of $z>1.3$ EROs are dusty star-forming galaxies.

This work is based on observations made with the Spitzer Space Telescope, which is operated by the Jet Propulsion Laboratory, California Institute of Technology, under NASA contract 1407. Support for this work was provided by NASA through contract 1256790 issued by JPL/Caltech. Support for the IRAC instrument was provided by NASA through contract 960541 issued by JPL. 
Alonso-Herrero, A., et al. 2004, ApJS, 154, 155

Bergström, S., \& Wiklind, T. 2004, A\&A, 414, 95

Chapman, S. C., Blain, A. W., Ivison, R. J., \& Smail, I. R. 2003, Nature, 422,695

Cimatti, A., et al. 2002a, A\&A, 381, L68 2002b, A\&A, 391, L1

Cowie, L. L., Gardner, J. P., Lilly, S. J., \& McLean, I. 1990, ApJ, 360, L1

Daddi, E., et al. 2003, ApJ, 588, 50

Davis, M., \& Geller, M. J. 1976, ApJ, 208, 13

Egami, E., Dole, H., \& Huang, J.-S. 2004, ApJS, 154, 130

Elbaz, D., Cesarsky, C. J., Chanial, P., Aussel, H., Franceschini, A., Fadda, D., \& Chary, R. R. 2002, A\&A, 384, 848

Elston, R., Rieke, G. H., \& Rieke, M. J. 1988, ApJ, 331, L77

Fazio, G. G., et al. 2004, ApJS, 154, 10

Franx, M., et al. 2003, ApJ, 587, L79

Graham, J. R., \& Dey, A. 1996, ApJ, 471, 720

Hasinger, G., et al. 2001, A\&A, 365, L45

Huang, J.-S., Barmby, P., Fazio, G. G., \& Wilner, S. 2004, ApJS, 154, 44

Huang, J.-S., Cowie, L. L., Gardner, J. P., Hu, E. M., Songaila, A., \& Wainscoat, R. J. 1997, ApJ, 476, 12

Huang, J.-S., et al. 2001, A\&A, 368, 787

Ivison, R. J., et al. 2004, ApJS, 154, 124

John, T. L. 1988, A\&A, 193, 189
REFERENCES

Le Floc'h, E., Pérez-González, P. G., Dole, H., \& Egami, E. 2004, ApJS, 154, 170

Mannucci, F., Pozzetti, L., Thompson, D., Oliva, E., Baffa, C., Comoretto, G., Gennari, S., \& Lisi, F. 2002, MNRAS, 329, L57

Miyazaki, S., et al. 2002, PASJ, 54, 833

Moustakas, L. A., et al. 2004, ApJ, 600, L131

Papovich, C., Dole, H., Egami, E., \& Pérez-González, P. G. 2004, ApJS, 154,70

Pozzetti, L., \& Mannucci, F. 2000, MNRAS, 317, L17

Rieke, G. H., Papovich, C., Dole, H., Egami, E., \& Pérez-González, P. G. 2004, ApJS, 154, 25

Roche, N. D., Almaini, O., Dunlop, J., Ivison, R. J., \& Willott, C. J. 2002, MNRAS, 337, 1282

Sawicki, M. 2002, AJ, 124, 3050

Scott, S. E., et al. 2002, MNRAS, 331, 817

Serjeant, S., et al. 2004, ApJS, 154, 118

Smail, I., Owen, F. N., Morrison, G. E., Keel, W. C., Ivison, R. J., \& Ledlow, M. J. 2002, ApJ, 581, 844

Somerville, R. S., Lee, K., Ferguson, H. C., Gardner, J. P., Moustakas, L. A., \& Giavalisco, M. 2004, ApJ, 600, L171

Somerville, R. S., et al. 2003, ApJ, 600, L135

Wilson, G. 2003, ApJ, 585, 191

Yan, L., \& Thompson, D. 2003, ApJ, 586, 765 